\title{
THE EFFECT OF LOW DOSE DOXYCYCLINE COMBINED WITH NONSURGICAL PERIODONTAL THERAPY ON THE LEVEL OF NITRIC OXIDE IN GINGIVAL CREVICULAR FLUID OF PATIENTS WITH STAGE I PERIODONTITIS. A RANDOMIZED CLINICAL TRIAL
}

\author{
Osama Mohamed Gouda* and Marwa Mohammed Tawfiq**
}

\begin{abstract}
The aim of this study was recording, the effect of conventional periodontal therapy (SRP) combined low dose doxycycline on nitric oxide level (NO) in gingival crevicular fluid (GCF) of patients with stage I periodontitis.
\end{abstract}

Materials and methods: 40 patients had stage I Periodontitis were grouped into two groups: (group 1): 20 patients will receive SRP at three sessions + doxycycline $20 \mathrm{mg}$ systemically twice daily (for 2 weeks). (Group 2): 20 patients will receive only SRP at three sessions for 2 weeks. With 20 healthy subjects acting as control group (group 3). Clinical evaluation and GCF sample was collected from two groups (1\&2) at base line, 15 and 30 days intervals and from healthy group at base line only.

Results: All the clinical indices and NO levels were found significantly higher in stage I Periodontitis groups compared to the control group at base line where $(\mathrm{p}<0.001)$ with no statistically significant differences between (Group 1) and (Group 2) where (p0.05>). When comparing the 2 studied patients groups and the healthy control group, founded improvement in clinical indices and NO levels at 15 and 30 days respectively with non-significant differences in all groups.

Conclusion: The current results provided that combined use SRP with low dose doxycycline had a reducing effect than SRP only for NO levels in GCF of patients with stage I periodontitis.

KEY WORDS: Grade I periodontitis; gingival crevicular fluid (GCF); low dose doxycycline; conventional periodontal therapy; nitric oxide (NO).

\footnotetext{
* Ass. Prof. Oral medicine, Periodontology, and Diagnosis, Faculty of Dentistry, Badr University.

** Lecturer of Oral medicine, Periodontology, and Diagnosis, Faculty of Oral and Dental Medicine Beni-Suef University.
} 


\section{INTRODUCTION}

Periodontitis is an inflammatory disease associated with multiple factors such as microbial agents found in the bacterial plaque, host susceptibility, and many environmental factors. The clinical features of periodontitis include gingival inflammation, clinical attachment loss (CAL), alveolar bone loss, periodontal pockets (PD), and gingival recession. (Flemmig, 1999, Kinane, 1999, Tonetti and Mombelli, 1999). A new periodontitis classification scheme has been adopted, in which forms of the disease previously recognized as "chronic" or "aggressive" are now grouped under a single category ("periodontitis") and are further characterized based on a multidimensional staging and grading system. Staging is largely dependent upon the severity of disease at presentation as well as on the complexity of disease management, while grading provides supplemental information about biological features of the disease including a history-based analysis of the rate of periodontitis progression; assessment of the risk for further progression; analysis of possible poor outcomes of treatment; and assessment of the risk that the disease or its treatment may negatively affect the general health of the patient. (Papapanou et al. 2018). The disease activity involves a network of interacting molecular pathways including proinflammatory mediators, reactive oxygen species (ROS), matrix metalloproteinase (MMP), and their MMP regulators and inhibitors (Birkedal-Hansen, 1993, Tervahartiala et al., 2000). Neutrophils (PMN) play a protective role in periodontal homeostasis, on the other side periodontal destruction has been associated with PMN hyperresponsivenes (Kinane et al., 2011 and Leppilahti et al., 2014). Reactive oxygen species (ROS) include a number of chemically reactive molecules derived from molecular oxygen emerged as important signaling molecules in regulation of various cellular processes. The most common free oxygen radicals include hydroxyl radical $(\mathrm{OH})$, nitric oxide $(\mathrm{NO})$, and superoxide $\left(\mathrm{O}_{2}^{-}\right)$. while the most common nonradical ROS is hydrogen peroxide $\left(\mathrm{H}_{2} \mathrm{O}_{2}\right)$ (Canakci et al., 2005). The antioxidant status and oxidative stress index are increased in patients with chronic periodontitis (Esen et al., 2012). The Nitric oxide (NO) is a one of ROS who plays an important role in increase pathogenesis of periodontal disease. It have extremely short life that cause fundamental damage to tissue and cellular components. The amount of tissue destruction can be assessed by measuring the levels of markers for lipid peroxidation, DNA damage, protein damage, and anti-oxidants (Dahiya et al., 2013).

Biomarkers of periodontal disease play an important role in diagnosis, assessing the treatment outcomes, and drug discovery. Among the important host-derived constituents in gingival crevicular fluid (GCF) are markers of inflammation, including enzymes, cytokines, and interleukins. Further, products of tissue breakdown can also be detected in crevicular fluid (Lamster and Ahlo, 2007, Teles et al., 2010, Grant et al., 2010, Dahiya et al., 2013).

In 2009, Tamaki et al., investigated the effect of non-surgical periodontal treatment on plasma reactive oxygen metabolites (ROMs) in patients with chronic periodontitis. At baseline, patients with chronic periodontitis had higher plasma ROM level than the control subjects. The clinical parameter showed a significant improvement 2 months after non-surgical periodontal treatment, and this was accompanied by a significant reduction in plasma ROM level. Chaudhary et al., in 2014 investigated the effect of surgical and non-surgical periodontal treatment on plasma ROM levels in chronic periodontitis $(\mathrm{CP})$ patients. In $\mathrm{CP}$ patients, surgical periodontal treatment was more effective in lowering the plasma ROM levels than when non-surgical periodontal treatment was performed alone.

A study was made to compare nitric oxide levels in stimulated whole saliva of individuals with and 
without chronic periodontitis. Nitric oxide levels was higher in chronic periodontitis group than healthy group (Reher et al., 2007). IN 2012, Parwani et al., estimated the salivary NO levels in inflammatory periodontal diseases (gingivitis and periodontitis) and comparing these levels with control subjects. NO levels were increased significantly in gingivitis and periodontitis individuals as compared with controls. There was a statistically significant decrease in the NO levels in each study group after the healing period (corresponding to the reduced clinical signs of inflammation).

Unstimulated whole saliva and serum samples were collected from a total of 60 subjects who participated in a study. They were divided into three equal groups with 20 subjects in each group; group A (healthy controls), group B (chronic periodontitis) and group $\mathrm{C}$ (aggressive periodontitis). A biochemical analysis was performed to evaluate and compare the salivary and the serum nitric oxide levels of the groups. There were statistically significant salivary and serum levels of NO in the groups of periodontitis (group B and C) as compared to those in the healthy controls (group A). A significant positive correlation was found between the values of the salivary and the serum NO levels in chronic and aggressive periodontitis (Sundar et al., 2013).

The most extensively investigated host modulatory agent is sub-antimicrobial dose of doxycycline (SDD) who widely used as adjunct to scaling and root planning (SRP). The therapeutic effect of SDD are executed via collagenase, osteoclast and cytokine inhibition in alveolar bone, connective tissue and epithelium (Burns et al., 1989, Gürkan et al., 2005, Sgolastra et al., 2011, Henehan et al., 2017). Doxycycline has the ability to scavenge radicals and reduce oxidative stress. One of the early published results demonstrated that doxycycline protected against peroxynitrite-induced degradation of $\alpha 1$-antiproteinase (Whiteman and
Halliwell, 1997). Other experiments showed that doxycycline showed radical scavenging capabilities (Leite et al.,2011) as well as inhibited the production of superoxide radical (Kładna et al., 2011). In addition, high concentrations of doxycycline may also directly react with superoxide and peroxynitrite in vitro and these findings corroborate the idea that doxycycline may be a potential ROS scavenger in hypertensive rats (Castro et al., 2012). Later investigations found that doxycycline decreased the oxidative stress index of gingival tissue in rats with periodontitis (Yağan et al., 2014).

A study on Adults with untreated chronic periodontitis were randomly administered to two groups, scaling and root planning and sub antimicrobial dose doxycycline (20 mg twice daily) or scaling and root planning +placebo treatment for 3 months. At baseline and after 3 months a gingivomucosal biopsy was collected to assay the induction of nitric oxide synthase (iNOS) and 3-nitrotyrosine (3NT), and blood was collected to assay for total nitrites and nitrates (NOx) and 3NT. The results compared to baseline, after the completion of treatment, significant decreases in the levels of tissue iNOS and 3NT and serum NOx and 3NT were observed in both groups. Scaling and root planning + sub antimicrobial dose doxycycline yielded greater reduction in the gingivomucosal and serum nitrosative stress markers than did scaling and root planning + placebo (Parvu et al., 2013). Clemens et al., 2018 confirmed that, one potential mechanism by which Doxycycline inhibits the progression of the periodontal diseases is by reducing oxidative stress, thereby inhibiting subsequent lipid peroxidation and inflammatory responses. This explains why it exhibits a clinical benefit beyond their antibacterial properties as it has direct and specific antioxidant properties. Finally, Moshira et al., 2019 showed more reduction in the nitric oxide level for chronic patients under SRP + systemic antimicrobial doxycycline treatment than chronic patients under SRP treatment only 
with insignificant differences in nitric oxide levels when compared to healthy control group at the end of the study period. The tenable explanation of these findings is attributed to the antioxidant and anti-proteolytic property effect of doxycycline. This study aimed to evaluate the effect of nonsurgical periodontal therapy combined with sub antimicrobial dose doxycycline on the nitric oxide levels in GCF of patients in stage I Periodontitis.

\section{SUBJECTS AND METHODS}

\section{Patient selection:}

40 Stage I Periodontitis patients with age range from 25-45 years were selected from the out patients clinic of the Oral Medicine, Oral Diagnosis and Periodontology Department - Minia University and Nahda University. The 40 patients will be grouped into two groups: group (1): 20 patients will receive non-surgical periodontal therapy consists of scaling and root planning (SRP) + doxycycline $20 \mathrm{mg}$ capsule twice daily* for 2 weeks. Group (2): 20 patients will receive only scaling and root planning (SRP) for 2 weeks. 20 healthy subjects will be included in the study with the same age range as a control group. A detailed medical history of each subject was obtained according to the detailed questionnaire of the modified Cornell Medical Index (Kerr and Millard 1965). All patients in this study must be systematically free, nonsmoker and no prior use of antibiotics in the last 6 months.

\section{Exclusion criteria}

1- Allergy to tetracycline

2- Female patients who are pregnant or of child bearing potential and not utilizing birth control.

3- Immunocompromised patients or those who are taking steroid medications.

\footnotetext{
*Vibramycin (Pfizzer company for medications)
}

\section{Ethical regulation}

The complete treatment plan will be explained to all patients including detailed steps, risks, and expected results and their full signed consent will be obtained prior to entry into study. The study will be complied with the rules set by the International Conference on Harmonization of Good Clinical Practice Guide lines, and the Declaration of Helsinki and the research ethics committee of the Faculty of Dentistry, Minia University.

\section{Clinical examination:}

Clinical parameters were recorded at baseline for each patient.

\section{Plaque Index (PI)}

The amount of dental plaque was scored according to the plaque index of (Silness and Loe, 1964).

\section{Gingival Index}

The degree of gingival inflammation was recorded by means of gingival index of (Löe and Silness, 1963), which divided tissues surrounding each tooth into four gingival scoring units: Disto -facial papilla, facial margin, mesio-facial papilla, and the entire lingual gingival margin.

\section{Probing depth (PD)}

Probing the pocket depth will be measured from the gingival margin to the base of the pocket (Glavind and Löe, 1967). It was measured using William's graduated periodontal probe.

\section{Probing Attachment level}

Probing the attachment level was measured from the cement-enamel junction (CEJ) to the base of the periodontal pocket (Glavind and Löe, 1967) using William's graduated periodontal probe. 


\section{Periodontal therapy:}

Two studied groups of Stage I Periodontitis was instructed to follow maintenance program for 2 weeks consists of brushing 2 times daily with soft tooth brush using plain tooth paste. Then, the two groups was subjected to non-surgical periodontal therapy compromising of three sessions of scaling and root planning for 2 weeks including full mouth supra and sub gingival scaling and root planning using hand instruments and ultrasonic scalers.

\section{Gingival crevicular fluid sample (GCF) collection}

The GCF sample was collected in the midmorning from two groups of Stage I Periodontitis to determine the inflammatory marker nitric oxide using the absorbent filter paper strips (Offenbacher et al. 1986) at baseline, 2 weeks and 1 month. The GCF sample was also collected from group 3 (healthy group) to determine nitric oxide marker at baseline only. The paper strips used is Whatman3MM Chromatography (Wh.3MM) strip. Using scalpel blades, chromatography papers were cut to the dimensions of $2 \times 8 \mathrm{~mm}$. The overall length of the paper was $12 \mathrm{~mm}$ greater than this to facilitate handling (Bogdanovska et al., 2017). The method of collection of GCF was intracrevicular (Loe and Holm-Pedersen, 1964). Sampling was done from the deepest probing site, taken from 3 sites and was stored in eppendorph tubes at $-20^{\circ} \mathrm{C}$ until be used for the laboratory analysis.

\section{V- Nitric oxide determination by Enzyme Linked Immunosorbant Assay (ELIZA):}

All collected samples of GCF from 3 groups were assayed using (ELISA) technique to assess the level of nitric oxide. The GCF from filter paper strips was diluted 1:2 - 1:20 into Reaction Buffer ultrafiltered through a 10,000 MWCO filter and used directly in the assay. The buffer used is deionized or distilled water. The Nitric Oxide Assay Kit (Assay Designs $^{\text {TM }}$ ) was used for quantitative determination of nitric oxide in biological fluids. Nitric oxide val- ues in the samples were recorded as in $\mu \mathrm{mol} / \mathrm{L}$ derived from the standard curve. Data were recorded as mean \pm standard deviation of the mean.

\section{Principle of the Assay: **}

This assay involved quantitative determination of nitric oxide in biological fluids. The kit involves the enzymatic conversion of nitrate to nitrite, by the enzyme nitrate reductase, followed by the colorimetric detection of nitrite as a colored azo dye product of the Griess reaction that absorbs visible light at $540 \mathrm{~nm}$.

\section{RESULTS}

\section{Comparison between groups:}

\section{A-Clinical Parameter}

\section{1-Plaque Index (PI)}

I- Baseline: A statistically significant difference was found between (Group 3) and each of (Group 1) and (Group 2) where $(p<0.001)$. No statistically significant difference was found between (Group 1) and (Group 2) where $(p<0.05)$.

II- Day 15: No statistically significant difference was found between (Groups 1 and 2) and healthy group where $(p<0.05)$.

III-Day 30: No statistically significant difference was found between all groups where $(p<0.05)$.

\section{2- Gingival Index (GI)}

I- Baseline: A statistically significant difference was found between (Group 3) and each of (Group 1) and (Group 2) where $(p<0.001)$. No statistically significant difference was found between (Group 1) and (Group 2) where $(\mathrm{p}<0.05)$.

II- Day 15: No statistically significant difference was found between (Groups 1 and 2) and healthy group where $(p<0.05)$. 
III-Day 30: No statistically significant difference was found between all groups where $(p<0.05)$.

\section{3- Probing pocket depth (PPD)}

I- Baseline: A statistically significant difference was found between (Group 3) and each of (Group 1) and (Group 2) where $(p<0.001)$. No statistically significant difference was found between (Group 1) and (Group 2) where $(p<0.05)$
II- Day 15: A statistically significant difference was found between (Group 3) and each of (Group 1) and (Group 2$)$ where $(p<0.001)$. No statistically significant difference was found between (Group 1) and (Group 2) where $(p<0.05)$.

III-Day 30: A statistically significant difference was found between (Group 3) and each of (Group 1) and (Group 2) where $(p<0.001)$. No statistically significant difference was found between (Group 1) and (Group 2) where $(p<0.05)$.

TABLE (1): The mean, standard deviation (SD) values of PI in each time period in different groups.

\begin{tabular}{|c|c|c|c|c|c|c|}
\hline \multirow{2}{*}{ Variables } & \multicolumn{4}{|c|}{ Plaque Index } \\
\cline { 2 - 7 } & \multicolumn{2}{|c|}{ Base Line } & \multicolumn{2}{c|}{ Day 15 } & \multicolumn{3}{c|}{ Day 30 } \\
\cline { 2 - 7 } & Mean & SD & Mean & SD & Mean & 0.13 \\
\hline Group 1 & 1.66 & 0.40 & 0.22 & 0.13 & 0.21 & 0.14 \\
\hline Group 2 & 1.63 & 0.45 & 0.23 & 0.45 & 0.15 & 0.02 \\
\hline Group 3 & 0.15 & 0.02 & 0.15 & 0.02 & 0.11 \\
\hline
\end{tabular}

TABLE (2): The mean, standard deviation (SD) values of GI in each time period in different groups.

\begin{tabular}{|c|c|c|c|c|c|c|}
\hline \multirow{2}{*}{ Variables } & \multicolumn{4}{|c|}{ Gingival Index } \\
\cline { 2 - 7 } & \multicolumn{2}{|c|}{ Base Line } & \multicolumn{2}{c|}{ Day 15 } & \multicolumn{3}{c|}{ Day 30 } \\
\cline { 2 - 7 } & Mean & SD & Mean & SD & Mean & 0.34 \\
\hline Group 1 & 1.70 & 0.45 & 0.20 & 0.33 & 0.20 & 0.23 \\
\hline Group 2 & 1.76 & 0.87 & 0.23 & 0.22 & 0.16 & 0.09 \\
\hline Group 3 & 0.16 & 0.09 & 0.16 & 0.09 & 0.22 \\
\hline
\end{tabular}

TABLE (3): The mean, standard deviation (SD) values of PD in each time period in different groups.

\begin{tabular}{|c|c|c|c|c|c|c|}
\hline \multirow{2}{*}{ Variables } & \multicolumn{4}{|c|}{ Probing pocket depth ( PPD ) } \\
\cline { 2 - 7 } & \multicolumn{2}{|c|}{ Base Line } & \multicolumn{2}{c|}{ Day 15 } & \multicolumn{2}{c|}{ Day 30 } \\
\cline { 2 - 7 } & Mean & SD & Mean & SD & 3.36 & 0.80 \\
\hline Group 1 & 3.95 & 0.81 & 3.47 & 0.88 & 3.40 & 0.77 \\
\hline Group 2 & 3.90 & 0.87 & 3.41 & 0.41 & 1.40 & 0.41 \\
\hline Group 3 & 1.40 & 0.41 & 1.40 & & 0.41 \\
\hline
\end{tabular}




\section{4- Clinical attachment level (CAL)}

I- Baseline: A statistically significant difference was found between (Group 3) and each of (Group 1) and (Group 2) where $(p<0.001)$. No statistically significant difference was found between (Group 1) and (Group 2) where $(p<0.05)$.

II- Day 15: A statistically significant difference was found between (Group 3) and each of (Group 1) and (Group 2) where $(p<0.001)$. No statistically significant difference was found between (Group 1) and (Group 2) where ( $p<005)$.

III-Day 30: A statistically significant difference was found between (Group 3) and each of (Group 1) and (Group 2$)$ where $(p<0.001)$. No statistically significant difference was found between (Group 1) and (Group 2) where $(p<0.05)$.

\section{B- Biological mediator (Nitric oxide):}

I- Baseline: A statistically significant difference was found between (Group 3) and each of (Group 1) and (Group 2) where $(p<0.001)$. No statistically significant difference was found between (Group 1) and (Group 2) where $(p<0.05)$.

II- Day 15: No statistically significant difference was found between (Group 3) and each of (Group 1) and (Group 2) where $(p<0.05)$. No statistically significant difference also was found between (Group 1) and (Group 2) where $(p<0.05)$.

III-Day 30: No statistically significant difference was found between (Group 3) and (two groups $1 \& 2)$ where $(p<0.05)$. Also no statistically significant difference was found between (Group 1) and (Group 2) where $(p<0.05)$.

TABLE (4): The mean, standard deviation (SD) values of CAL in each time period in different groups.

\begin{tabular}{|c|c|c|c|c|c|c|}
\hline \multirow{2}{*}{ Variables } & \multicolumn{9}{|c|}{ Clinical attachment level ( CAL ) } \\
\cline { 2 - 7 } & \multicolumn{2}{|c|}{ Base Line } & \multicolumn{2}{c|}{ Day 15 } & \multicolumn{3}{c|}{ Day 30 } \\
\cline { 2 - 7 } & Mean & SD & Mean & SD & Mean & SD \\
\hline Group 1 & 1.35 & 0.91 & 1.26 & 0.96 & 1.25 & 0.79 \\
\hline Group 2 & 1.43 & 0.87 & 1.41 & 0.85 & 0.70 \\
\hline Group 3 & 0.00 & 0.00 & 0.00 & 0.00 & 0.00 & 0.00 \\
\hline
\end{tabular}

TABLE (5): The mean, standard deviation (SD) values of Nitric Oxide in each group.

\begin{tabular}{|c|c|c|c|c|c|c|}
\hline \multirow{2}{*}{ Variables } & \multicolumn{4}{|c|}{ Nitric-Oxide } & \multicolumn{2}{c|}{ Day 30 } \\
\cline { 2 - 7 } & \multicolumn{2}{|c|}{ Base Line } & \multicolumn{2}{c|}{ Day 15 } & Mean & SD \\
\cline { 2 - 7 } & Mean & SD & Mean & SD & 2.84 & 0.35 \\
\hline Group 1 & 7.45 & 0.70 & 2.82 & 0.33 & 3.95 & 0.95 \\
\hline Group 2 & 7.77 & 0.85 & 3.94 & 1.30 & 2.58 & 0.38 \\
\hline Group 3 & 2.58 & 0.38 & 2.58 & 0.38 & \\
\hline
\end{tabular}




\section{DISCUSSION}

The human periodontal diseases are inflammatory disorders that cause tissue damage and loss, as a result of the complex interaction between pathogenic bacteria and the host immune response accompanied by an increase in cytokine expression and immunological activity in gingival tissues. Thus PMN production of large amount of ROS. The Nitric oxide is one of oxygen radical of ROS that play a role in pathogenesis of periodontitis (BirkedalHansen, 1993, Lohinai et al., 1998 and Canakci et al., 2005).

Several studies have investigated the role of NO in the progression of periodontal diseases (Amine., et al., 1996, Battino., et al 1999, Ali., et al., 2014 and Moshira et. Al., 2019).

This study aimed to evaluate the effect of non-surgical periodontal therapy combined with sub antimicrobial dose doxycycline on the level of nitric oxide in GCF of patients affected by stage I periodontitis. Whiteman and Halliwell, 1997 demonstrated that doxycycline protected against peroxynitrite-induced degradation of $\alpha 1$ antiproteinase. Another investigation suggested that doxycycline decreased the oxidative stress index of gingival tissue in rats with periodontitis (Yağan et al., 2014). The flow of gingival crevicular fluid is an important determinant for the status of periodontal tissues. Reports have found that several crevicular biomarkers could be used to detect and/ or predict periodontal disease activity (Souza and Taba Jr, 2004). It has been previously founded that the nitric oxide (NO) levels in gingival crevicular fluid can be used as early detection marker in periodontal inflammation (Ali et al., 2014).

The present study employed non-surgical periodontal therapy due to its an efficient method for reduction of the gingival inflammation, reduction of probing pocket depth, and gain in clinical attachment level (Bogdanovska et al., 2017). The present study was performed to measurement of nitric oxide levels in gingival crevicular fluid rather than serum as gingival crevicular fluid has the benefit of atraumatic technique, enables repeated sampling at different times from the same site, being closely approximated to the site of destruction, and thus provide more information. Therefore, this study was conducted in accordance with the study which carried out by Esen et al., 2012. The absorbent filter paper strips was used in this study as it is less invasive and the least traumatic technique. Whatman 3MM chromatography paper (Wh.3MM) was used according to the work of Bogdanovska et al., 2017. In order to limit the potential effects of proteases from the host and bacterial sources, samples were kept cold immediately after sample collection and quickly transferred to be stored in -20 for analysis. The substance contained in the filter paper was extracted prior to analysis. There is growing evidence of the involvement of nitric oxide (NO) and nitro-oxidative stress in chronic periodontitis that has highlighted the need to improve diagnostic and treatment algorithms (Menaka et al., 2009). NO is produced in vivo from $L$-arginine by nitric oxide synthase (NOS). nNOS/ NOS1 and eNOS/NOS3 are constitutive isoforms of the enzyme found in neuronal and endothelial cells. Inducible NOS (NOS2/iNOS) is expressed by various cells, such as macrophages and fibroblasts, in response to inflammatory stimuli, and it produces large amounts of NO. NO has been implicated in the pathophysiology of several inflammatory conditions such as periodontal disease since production of large amount of NO are toxic ( Menaka et al., 2009).

Several studies showed that oxidative stress is induced by an imbalance between excessive ROS production and anti-oxidant mechanisms. Increased levels of NO leads to a state of Oxidative Stress have been implicated in the pathogenesis of periodontitis (Sapadin and Fleischmajer, 2006 \& Chapple and Matthews, 2007).

The pathogenic nitric oxide synthase (NOS) has been decreased by doxycycline. The antioxidant effect of doxycycline does not originate from its capability to directly scavenge ROS but from its 
influence on white blood cells especially neutrophils (Miyachi et al., 1986).

In the present study Vibramycin has been used in adjunct to non-surgical periodontal therapy in one group and the other group received only nonsurgical periodontal therapy in order to show the effect of doxycycline as antioxidant to nitric oxide which is involved in pathogenesis of periodontal disease. Doxycycline was used systemically as $20 \mathrm{mg}$ oral capsule twice daily for two weeks. It combats oxidative stress, mop up free radicals and inhibit an excessive inflammatory response secondary to an antigenic stimulus (Brock et al., 2004 \& Sapadin and Fleischmajer, 2006).

The present results demonstrated that group I and group II showed decrease in nitric oxide level that where statistically significant when compared to baseline. When comparing the two groups the decrease in nitric oxide level were more favorable in group I as compared to group II at each follow up period. Significant reduction in GI and PI at follow up period day 15 and day30 was recorded in both group1 and group2.This may be attributed to the comprehensive treatment modality and oral hygiene maintenance that offered coverage of the major etiologic factors of both groups. Furthermore, statistical analysis of data showed non-significant differences in the clinical parameters between the two groups at day 15 and day30 after treatment as regard CAL and PD compared to baseline. When comparing group I and group II, the decrease in PD was more favorable in group I as compared to group I at follow up period day30 .This is attributed to anti-collagenase property of tetracycline (Chang et al., 2015).

Interstitial collagenases are proteinase-type enzymes, these enzymes are derived from a number of sources including fibroblast, epithelial cells, macrophages (MMP-1), and neutrophils (MMP-8). Tetracycline is less active against fibroblast-type collagenase and most active against neutrophilderived collagenase. Doxycycline is the most potent tetracycline for collagenase inhibition. It has been found that these oxygen radicals activate latent collagenase, thus tetracycline can prevent the oxidative activation of latent collagenase (Golub et al., 1991).

In the present study, nitric oxide level was reduced in both groups (group I and group II) after day 15 and day30. There was insignificant difference between group I and group II at baseline, on the other hand group I showed more reduction in the nitric oxide level than group II at follow up period day 15 and day30. Also group I showed insignificant difference in nitric oxide level when compared to group III (healthy control) at day 15 and day30, the tenable explanation of these findings is attributed to the antioxidant effect of doxycycline. The significant favorable decrease in nitric oxide level from baseline to day30 in group I suggests the role doxycycline as antioxidant. This may be related to the ability of doxycycline to scavenge reactive oxygen radicals produced by PMNs. It has been found that these oxygen radicals activate latent collagenase, thus doxycycline can prevent the oxidative activation of latent collagenase (Da Silva, et al., 2018).

The anti-proteolytic property, doxycycline inhibition of neutrophil collagenase may also prevent other proteolytic events because neutrophil collagenase (MMP-8) as well as neutrophil-derived reactive oxygen species, i.e., hydrogen peroxide, and nitric oxide can degrade and inactivate $\alpha-1$ proteinase inhibitor (Rai et al., 2010 and Moshira et al., 2019).

\section{CONCLUSION}

The current investigation detected that use of scaling and root planning (SRP) combined with sub antimicrobial dose of doxycycline systemically had more effective than scaling and root planning (SRP) only in reducing the nitric oxide levels in gingival crevicular fluid (GCF) of patients with stage I periodontitis. 


\section{REFERENCES}

1. Ali, O. T., Akalin, F. A., Sahbazoglu, K. B., Yamalik, N., Kilinc, K., Karabulut, E. \& Tözum, T. F. 2014. Nitrite and nitrate levels of gingival crevicular fluid and saliva in subjects with gingivitis and chronic periodontitis. Journal of oral \& maxillofacial research, 5.

2. Amin, A. R., Attur, M. G., Thakker, G. D., Patel, P. D., Vyas, P. R., Patel, R. N., Patel, I. R. \& Abramson, S. B. 1996. A novel mechanism of action of tetracyclines: effects on nitric oxide synthases. Proceedings of the National Academy of Sciences, 93, 14014-14019.

3. Battino, M., Bullon, P., Wilson, M. \& Newman, H. 1999. Oxidative injury and inflammatory periodontal diseases: the challenge of anti-oxidants to free radicals and reactive oxygen species. Critical Reviews in Oral Biology \& Medicine, 10, 458-476.

4. Birkedal-Hansen, H. 1993. Role of matrix metalloproteinases in human periodontal diseases. Journal of periodontology, 64, 474-484.

5. Birkedal-Hansen, H. 1993. Role of cytokines and inflammatory mediators in tissue destruction. Journal of periodontal research, 28, 500-510.

6. Bogdanovska, L., Panovska, A., Popovska, M., Dimitrovska, A. \& PETKOVSKA, R. 2017.

7. Chemometric Evaluation Of The Efficacy Of Locally Administered Chlorhexidine In Patients With Periodontal Disease. Saudi pharmaceutical journal, 25, 1022-1031.

8. Brill, N. \& Krasse, B. 1958. The passage of tissue fluid into the clinically healthy gingival pocket. Acta Odontologica Scandinavica, 16, 233-245.

9. Brock, G., Butterworth, C., Matthews, J. \& Chapple, I. 2004. Local and systemic total antioxidant capacity in periodontitis and health. Journal of Clinical Periodontology, 31, 515-521.

10. Burns, F., Stack, M., Gray, R. \& Paterson, C. 1989. Inhibition of purified collagenase from alkali-burned rabbit corneas. Investigative ophthalmology \& visual science, 30, 1569-1575.

11. Canakci, C., Cicek, Y. \& Canakci, V. 2005. Reactive oxygen species and human inflammatory periodontal diseases. Biochemistry (Moscow), 70, 619-628.

12. Castro, M. M., Rizzi, E., Ceron, C. S., Guimaraes, D. A., Rodrigues, G. J., Bendhack, L. M., Gerlach, R. F. \& Tanus-
Santos, J. E. 2012. Doxycycline ameliorates 2K-1C hypertension-induced vascular dysfunction in rats by attenuating oxidative stress and improving nitric oxide bioavailability. Nitric Oxide, 26, 162-168.

13. Chang, R., Raoufinia, A., \& Shaha, N. 2015. Once daily formulations of tetracyclines. Google patents.

14. Chapple, I. L. \& Matthews, J. B. 2007. The role of reactive oxygen and antioxidant species in periodontal tissue destruction. Periodontology 2000, 43, 160-232.

15. Chaudhary, S., Gowda, T. M., Mehta, D. S. \& Kumar, T. A. 2014. Comparative evaluation of plasma ROM levels in chronic periodontitis patients before and after non-surgical and surgical periodontal therapy: A clinical trial. Journal of Indian Society of Periodontology, 18, 140.

16. Clemens, D., Duryee, M., Sarmiento, C., Chiou, A., Mcgowan, J., Hunter, C., \& O'dell, J. 2018. Novel antioxidant properties of Doxycucline. international journal of molecular sciences, 19, 4078.

17. Dahiya, P., Kamal, R., Gupta, R., Bhardwaj, R., Chaudhary, K. \& Kaur, S. 2013. Reactive oxygen species in periodontitis. Journal of Indian Society of Periodontology, 17, 411 .

18. Da Silva, J, Muniz, F, Oballe, H, \& Cavagni, J, 2018. The effect of periodontal therapy on oxidative stress biomarkers: A systemic review. Journal of clinical periodontology. $1237-1222,45$.

19. Esen, Ç., Alkan, B. A., Kırnap, M., Akgul, Ö., Işıkoğlu, S. \& Erel, Ö. 2012. The effects of chronic periodontitis and rheumatoid arthritis on serum and gingival crevicular fluid total antioxidant/oxidant status and oxidative stress index. Journal of periodontology, 83, 773-779.

20. Flemmig, T. F. 1999. Periodontitis. Ann Periodontol, 4, 32-8.

21. Glavind, L. \& Löe, H. 1967. Errors in the clinical assessment of periodontal destruction. Journal of periodontal Research, 2, 180-184.

22. Golub, L. M., Ramamurthy, N., Mcnamara, T. F., Greenwald, R. A. \& Rifkin, B. R. 1991. Tetracyclines inhibit connective tissue breakdown: new therapeutic implications for an old family of drugs. Critical Reviews in Oral Biology \& Medicine, 2, 297-321.

23. Grant, M. M., Brock, G. R., Matthews, J. B. \& Chapple, I. L. 2010. Crevicular fluid glutathione levels in periodontitis and the effect of non-surgical therapy. Journal of clinical periodontology, 37, 17-23. 
24. Gurkan, A., Çınarcık, S. \& Huseyinov, A. 2005. Adjunctive subantimicrobial dose doxycycline: effect on clinical parameters and gingival crevicular fluid transforming growth factor- $\beta 1$ levels in severe, generalized chronic periodontitis. Journal of clinical periodontology, 32, 244253.

25. Henehan, M., Montuno, M. \& De Benedetto, A. 2017. Doxycycline As An Anti-Inflammatory Agent: Updates in Dermatology. Journal of the European Academy of Dermatology and Venereology.

26. Kerr D A., and Millard H D.: Oral Diagnosis, 1965; $2^{\text {nd }}$ edition

27. Kinane, D. F. 1999. Periodontitis modified by systemic factors. Annals of Periodontology, 4, 54-63.

28. Kinane, D. F., Preshaw, P. M. \& Loos, B. G. 2011. Hostresponse: understanding the cellular and molecular mechanisms of host-microbial interactions--consensus of the Seventh European Workshop on Periodontology. J Clin Periodontol, 38 Suppl 11, 44-8.

29. Kładna, A., Kruk, I., Michalska, T., Berczyński, P. \& Aboul-Enein, H. Y. 2011. Characterization of the superoxide anion radical scavenging activity by tetracycline antibiotics in aprotic media. Luminescence, 26, 611-615.

30. LAMSTER, I. B. \& AHLO, J. K. 2007. Analysis of gingival crevicular fluid as applied to the diagnosis of oral and systemic diseases. Annals of the New York Academy of Sciences, 1098, 216-229.

31. Leite, L. M., Carvalho, A. G., Ferreira, P. L., Pessoa, I. X., Goncalves, D. O., Lopes Ade, A., Goes, J. G., Alves, V. C., Leal, L. K., Brito, G. A. \& Viana, G. S. 2011. Antiinflammatory properties of doxycycline and minocycline in experimental models: an in vivo and in vitro comparative study. Inflammopharmacology, 19, 99-110.

32. Leppilahti, J. M., Hernandez-Rios, P. A., Gamonal, J. A., Tervahartiala, T., Brignardello-Petersen, R., Mantyla, P., Sorsa, T. \& Hernandez, M. 2014. Matrix metalloproteinases and myeloperoxidase in gingival crevicular fluid provide site-specific diagnostic value for chronic periodontitis. J Clin Periodontol, 41, 348-56.

33. Loe, H. \& Holm-Pedersen, P. 1964. Absence and presence of fluid from normal and inflamed gingivae. Periodontics, 3, 171-177.

34. LöE, H. \& SILNESS, J. 1963. Periodontal disease in pregnancy I. Prevalence and severity. Acta odontologica scandinavica, 21, 533-551.
35. Lohinai, Z., Benedek, P., Feher, E., Györfi, A., Rosivall, L., Fazekas, Á., Salzman, A. L. \& Szabó, C. 1998. Protective effects of mercaptoethylguanidine, a selective inhibitor of inducible nitric oxide synthase, in ligature-induced periodontitis in the rat. British journal of pharmacology, 123, 353-360.

36. Menaka, K., Ramesh, A., Thomas, B. \& Kumari, N. S. 2009. Estimation of nitric oxide as an inflammatory marker in periodontitis. Journal of Indian Society of Periodontology, 13,75 .

37. Miyachi, Y., Yoshioka, A., Imamura, S. \& Niwa, Y. 1986. Effect of antibiotics on the generation of reactive oxygen species. J Invest Dermatol, 86, 449-53.

38. Moshira, A., Mohamed, A., Osama, M., \& Ahmed, A. 2019.

39. Effect of non-surgical periodontal therapy combined with doxycycline on the levels of gingival crevicular fluid of nitric oxide. Egyptian dental J. Vol. 65, 1:6 October.

40. Offenbacher S, Odle BM, Van Dyke TE: The use of crevicular fluid prostaglandin E2 levels as a predictor of periodontal attachment loss. J Periodontal RES. 1986; 21:101-12.

41. Papapanou et al. 2018. Periodontitis: Consensus report of workgroup 2 of the 2017World Workshop on the Classification of Periodontal and Peri-Implant Diseases and Conditions. Journal of Periodontol;89(Suppl 1):S173-S182.

42. Parvu, A. E., Alb, S. F., Crăciun, A. \& Taulescu, M. A. 2013. Efficacy of subantimicrobial-dose doxycycline against nitrosative stress in chronic periodontitis. Acta Pharmacologica Sinica, 34, 247-254.

43. Parwani, S., Chitnis, P. \& Parwani, R. 2012. Salivary nitric oxide levels in inflammatory periodontal disease-A casecontrol and interventional study. International journal of dental hygiene, 10, 67-73.

44. Rai, B., Kaur, J., Jain, R. \& Anand, S., 2010. Levels of gingival crevicular metalloproteinases-8 and -9 in periodontitis. The Saudi dental journal 22, 129-131.

45. Reher, V. G., Zenóbio, E. G., Costa, F. O., Reher, P. \& Soares, R. V. 2007. Nitric oxide levels in saliva increase with severity of chronic periodontitis. Journal of oral science, 49, 271-276.

46. Sapadin, A. N. \& Fleischmajer, R. 2006. Tetracyclines: nonantibiotic properties and their clinical implications. $\mathrm{J}$ Am Acad Dermatol, 54, 258-65.

47. Sgolastra, F., Petrucci, A., Gatto, R., Giannoni, M. \& Monaco, A. 2011. Long-term efficacy of subantimicrobialdose doxycycline as an adjunctive treatment to scaling and 
root planing: a systematic review and meta-analysis. Journal of periodontology, 82, 1570-1581.

48. Silness, J. \& Loe, H. 1964. Periodontal Disease In Pregnancy. Ii. Correlation Between Oral Hygiene And Periodontal Condtion. Acta Odontol Scand, 22, 121-35.

49. Souza, S. L. S. D. \& Taba Jr, M. 2004. Cross-sectional evaluation of clinical parameters to select high prevalence populations for periodontal disease: the site comparative severity methodology. Brazilian dental journal, 15, 46-53.

50. Sundar, N. M., Krishnan, V., Krishnaraj, S., Hemalatha, V. \& Alam, M. N. 2013. Comparison of the salivary and the serum nitric oxide levels in chronic and aggressive periodontitis: a biochemical study. Journal of clinical and diagnostic research: JCDR, 7, 1223.

51. Tamaki, N., Tomofuji, T., Ekuni, D., Yamanaka, R., Yamamoto, T. \& Morita, M. 2009. Short-term effects of nonsurgical periodontal treatment on plasma level of reactive oxygen metabolites in patients with chronic periodontitis. Journal of periodontology, 80, 901-906.
52. Teles, R., Sakellari, D., Teles, F., Konstantinidis, A., Kent, R., Socransky, S. \& Haffajee, A. 2010. Relationships among gingival crevicular fluid biomarkers, clinical parameters of periodontal disease, and the subgingival microbiota. Journal of periodontology, 81, 89-98.

53. Tervahartiala, T., Pirilä, E., Ceponis, A., Maisi, P., Salo, T., Tuter, G., Kallio, P., Törnwall, J., Srinivas, R. \& Konttinen, Y. 2000. The in vivo expression of the collagenolytic matrix metalloproteinases (MMP-2,-8,-13, and-14) and matrilysin (MMP-7) in adult and localized juvenile periodontitis. Journal of dental research, 79, 1969-1977.

54. Tonetti, M. S. \& Mombelli, A. 1999. Early-onset periodontitis. Annals of Periodontology, 4, 39-52.

55. Whiteman, M. \& Halliwell, B. 1997. Prevention of peroxynitrite-dependent tyrosine nitration and inactivation of alpha1antiproteinase by antibiotics. Free Radic Res, 26,49-56.

56. Yağan, A., Kesim, S. \& Liman, N. 2014. Effect of low-dose doxycycline on serum oxidative status, gingival antioxidant levels, and alveolar bone loss in experimental periodontitis in rats. Journal of periodontology, 85, 478-489. 